\title{
Safety, Feasibility, and Results of Exercise Testing for Stratifying Patients with Chest Pain in the Emergency Room
}

\author{
Renato Machado Macaciel, Evandro Tinoco Mesquita, Ricardo Vivacqua, Salvador Serra, \\ Augusta Campos, Marcelo Miranda, Roberto Gamarski, Hans Dohman, Roberto Bassan, \\ and the research team of the Chest Pain Project
}

Rio de Janeiro, RJ - Brazil

\begin{abstract}
Objective - To assess safety, feasibility, and the results of early exercise testing in patients with chest pain admitted to the emergency room of the chest pain unit, in whom acute myocardial infarction and high-risk unstable angina had been ruled out.
\end{abstract}

Methods - A study including 1060 consecutive patients with chest pain admitted to the emergency room of the chest pain unit was carried out. Of them, $677(64 \%)$ patients were eligible for exercise testing, but only 268 (40\%) underwent the test.

Results - The mean age of the patients studied was $51.7 \pm 12.1$ years, and $188(70 \%)$ were males. Twenty-eight (10\%) patients had a previous history of coronary artery disease, $244(91 \%)$ had a normal or unspecific electrocardiogram, and $150(56 \%)$ underwent exercise testing within a 12-hour interval. The results of the exercise test in the latter group were as follows: 34 (13\%) were positive, 191 (71\%) were negative, and $43(16 \%)$ were inconclusive. In the group of patients with a positive exercise test, 21 (62\%) underwent coronary angiography, 11 underwent angioplasty, and 2 underwent myocardial revascularization. In a univariate analysis, type A/B chest pain (definitely/probably anginal) ( $p<0.0001)$, previous coronary artery disease $(p<0.0001)$, and route 2 (patients at higher risk) correlated with a positive or inconclusive test ( $p<0.0001)$.

Conclusion - In patients with chest pain and in whom acute myocardial infarction and high-risk unstable angina had been ruled out, the exercise test proved to be feasible, safe, and well tolerated.

Keywords: exercise test, chest pain, emergency room, chest pain unit

Hospital Pró-Cardíaco and Universidade Federal Fluminense Mailing address: Renato M. Macaciel - Hospital Pró-Cardíaco - Rua Dona Mariana, 219-22280-020 - Rio do Janeiro, RJ, Brazil -E-mail: macaciel@ cardiol.br English version by Stela Maris C. e Gandour
Chest pain is one of the most common reasons for seeking medical attention in the emergency department. In the United States, approximately 5 million symptomatic emergency assistances are estimated to occur, corresponding to approximately 5 to $10 \%$ of the total assistance ${ }^{1,2}$. In our country, data from Datasus show that, in the first half of the 1990 s, coronary artery disease accounted for approximately $30 \%$ of the total of deaths ${ }^{3,4}$. Patients presenting with a complaint of chest pain are usually a diagnostic challenge, because several chest disorders may cause the symptom, including those with a high mortality rate, such as acute myocardial infarction. However, only 10 to $15 \%$ of the pa tients presenting with chest pain have acute myocardial infarction, and less than $1 \%$ have pulmonary embolism or aortic dissection. However, more than $50 \%$ of those patients are hospitalized for an extensive and expensive diagnostic investigation $^{5-7}$.

An initial 12-lead electrocardiogram is diagnostic in only $50 \%$ of the patients with chest pain and acute myocardial infarction. A single measurement of CPK-MB has a $35 \%$ sensitivity to detect infarction ${ }^{8}$. In addition, approximately 2 to $5 \%$ of the patients admitted to the emergency department with the cited symptoms are inadvertently discharged from the hospital without a diagnosis, which results in an increase in morbidity and mortality. The complications of these patients, including death, result in $20 \%$ of the lawsuits due to medical malpractice in the USA ${ }^{9,10}$, and they may be found in young patients with atypical chest pain and normal or nondiagnostic electrocardiograms.

Exercise testing is a widely available and inexpensive method, a well-studied diagnostic and prognostic tool for chronic coronary artery disease. However, its role in the diagnostic and prognostic stratification of patients with chest pain and with an uncertain diagnosis in the emergency department has not been well established.

Since the beginning of the 1990s, exercise testing has been used to stratify very low-risk symptomatic patients ${ }^{11-13}$. With the appearance of systematic strategies for the assis- 
tance of these patients in chest pain units and the inclusion of myocardial necrosis markers, enabling more safely ruling out the possibility of acute myocardial infarction and highrisk unstable angina, the method has been used in patients with a low to intermediate probability of acute myocardial infarction and low risk for cardiac events. The method has also been extended to patients with a previous history of coronary artery disease and intermediate- and low-risk unstable angina after clinical stabilization ${ }^{14}$. At the Hospital Pró-Cardíaco, exercise testing began to be used in a pioneering way in Brazil in patients with low-risk chest pain ${ }^{15}$.

Our objective was to assess the safety, feasibility, and the results of early exercise testing in emergency department chest pain patients, in whom acute myocardial infarction and high-risk unstable angina had been ruled out.

\section{Methods}

A prospective cohort study was carried out in 1060 patients assisted at the emergency room of the chest pain unit of a tertiary cardiology hospital from November 1996 to May 1998. The patients had nontraumatic acute chest pain lasting less than 12 hours.

The patients underwent a diagnostic evaluation protocol (Chest Pain Project). At first, the type of chest pain and the characteristics of the electrocardiogram on hospital admission were assessed. Then, patients were assigned to diagnostic strategies or routes, in which procedures of diagnostic and prognostic determination and therapeutic management were predetermined (measurement of myocardial necrosis markers, chest radiography, and echocardiography), and, finally, they were referred or not for exercise testing.

The following patients were excluded from the study: patients with acute myocardial infarction; with elevation in the ST segment; with type A/B chest pain and depression in the ST segment or inversion in the T wave, or both; and with left bundle-branch block and type D2 chest pain (noncardiac).

The chest pain was classified into 4 types: type A, definitively anginal chest pain; type $B$, probably anginal chest pain; type $\mathrm{C}$, probably nonanginal chest pain; type $\mathrm{D}$, definitively nonanginal chest pain (subdivided into 2 subgroups: D1 - without a high suspicion of another diagnosis, and D2 - with a high suspicion of another diagnosis).

After classifying the type of chest pain, the 18-lead electrocardiogram ( 12 conventional plus 4 right precordial plus 2 dorsal leads) was performed at 3 -hour intervals until 9 hours (0-3-6-9 hours), according to the route assigned. The electrocardiograms were classified into 4 types as follows: 1 ) with elevation of the ST segment; 2) with depression of the ST segment or inversion of the T wave; 3 ) with left bundlebranch block; 4) normal or unspecific ${ }^{16}$.

The patients with chest pain and electrocardiograms strongly suggestive of acute myocardial infarction referred to the coronary unit were assigned to route 1 . The patients with electrocardiographic changes in depression of the J-ST segment or inversion of the T wave, or those with type A/B chest pain but with no electrocardiographic alterations indicating myocardial ischemia were assigned to route 2 . Due to the high likelihood of having unstable angina and the medium likelihood of having acute myocardial infarction, these patients remained in the emergency department, where they underwent serial electrocardiographic and enzymatic evaluations ( $3 / 3$ hours) and echocardiography in the following 9 hours. The patients with chest pain whose characteristics did not allow complete exclusion of acute coronary heart disease (type C) and with no electrocardiographic changes in myocardial ischemia were assigned to route 3 . Due to the low likelihood of having unstable angina and acute myocardial infarction, these patients also remained in the emergency department, where they underwent serial electrocardiographic and enzymatic evaluations ( $3 / 3$ hours) in the following 6 hours. Those patients assigned to route 2 who were asymptomatic and had no recurring ischemia were referred for exercise testing (within 24 to 48 hours). On the other hand, patients initially assigned to route 3 and with no myocardial ischemia during the evaluation period in the emergency department underwent exercise testing within 9 to 12 hours after admission.

The patients with chest pain considered nonanginal (type D) and with no electrocardiographic changes indicating myocardial ischemia were assigned to route 4 . They underwent exercise testing immediately or were discharged with the recommendation to undergo exercise testing later (fig. 1).

In this study, CK-MB and its relation to $\mathrm{CPK}$, markers of myocardial necrosis, were considered references for the diagnosis of myocardial necrosis. Measurements were performed at 3-hour intervals until 9 hours according to the route assigned ${ }^{16}$.

The study was approved by the committee on ethics and research of the Hospital Pró-Cardíaco.

Exercise testing was performed in a laboratory connected to the emergency department by using analysis of 13 leads with the modified lead system of Mason and Likar ${ }^{17}$, with CM5 (a lead similar to V5), in a Micromed computerized device and Ibramed treadmill. Most protocols used were by Bruce et al ${ }^{18}$ or adapted to the biomechanical conditions of the patients, according to the physician responsible for the examination, and always symptom-limited. After undergoing the examination, the patient returned to the emergency department, where the route assigned and the result of the test were analyzed. The patient, then, was discharged or admitted to the hospital to proceed with the investigation. The following diagnostic parameters of the exercise test were associated with myocardial ischemia: development of angina during or immediately after exertion; depression of the ST segment measured from point $Y(80 \mathrm{~ms}$ from point $\mathrm{J}) \geq 1 \mathrm{mV}$, with horizontal or descending ST segments; depression of the ST segment measured from point $Y(80 \mathrm{~ms}$ from point $\mathrm{J})$ $\geq 0.15 \mathrm{mV}$ for males and $0.2 \mathrm{mV}$ for females, with a slow ascending ST segment; elevation of the ST segment measured $80 \mathrm{~ms}$ from point $\mathrm{J} \geq 1 \mathrm{mV}$ in a lead with no pathological Q 


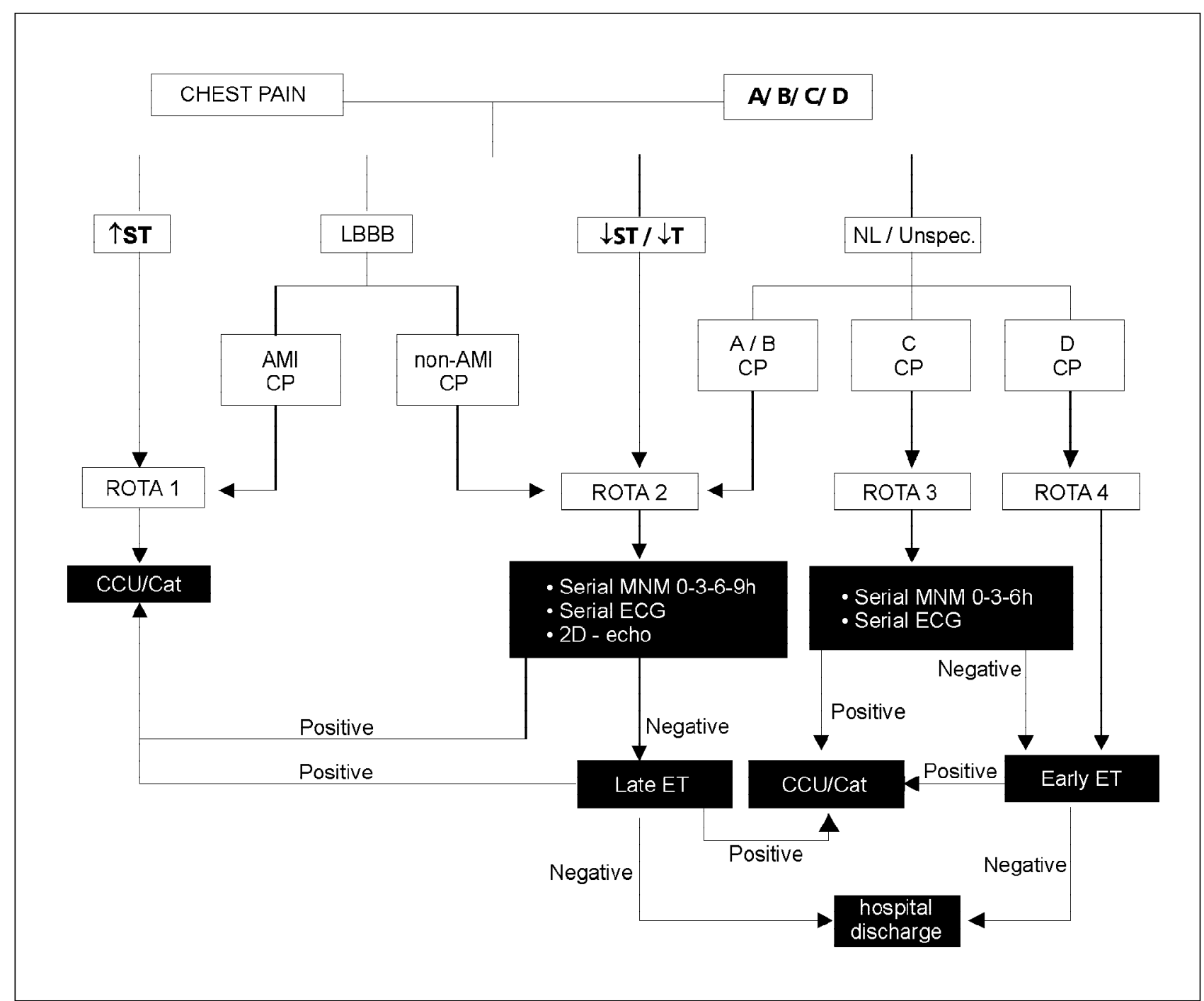

Fig.1 - Flowchart of assistance in the chest pain unit. ET- exercise test; CCU- coronary care unit; MNM- myocardial necrosis markers; NL- normal; LBBB- left bundle-branch block; CAT- cine coronary angiography; ECHO 2D- echocardiography; CP- chest pain

wave; depressed response of or drop in heart rate during exercise (in the presence of ischemic changes in the ST segment), when the patient was not receiving beta-blockers; a plateau or drop in systolic blood pressure $>10 \mathrm{mmHg}$ (as compared with the previous stage or rest) on exertion associated with ischemic changes in the ST segment.

According to the result, the exercise tests were classified as: positive - in the presence of the criteria for positivity: anginal pain or changes in the ST segment or hemodynamic changes, or both; negative - in the absence of ischemia, with heart rate > submaximal; inconclusive - when the patient did not reach the heart rate required ( $85 \%$ of the previewed maximal heart rate), receiving or not beta-blockers, in the absence of ischemic changes on the electrocardiogram or angina.

The statistical analysis was performed as follows: 1) in the comparison of the middle levels of the 3 groups of patients (ex: positive, negative, and inconclusive), the analysis of variance (ANOVA) or the Kruskal-Wallis test was used. The Tukey test and the multiple comparisons test based on Kruskal-Wallis statistics were used to identify the groups differing amongst themselves; 2 ) for comparing the means between 2 independent groups, the Student $t$ test or the Mann-Whitney test was used; 3 ) for comparing the proportions, the chi-square test or Fisher exact test was used. The significance level adopted was 5\%.

\section{Results}

From November 1996 to May 1998, 1060 consecutive patients with chest pain were admitted to the emergency room of the chest pain unit and included in the assessment protocol after mutual consent of the patient and the assistant physician. Their mean age was $59 \pm 15.3$ years, and males prevailed (62.7\%). Almost half of the patients had type A/B chest pain, $43.4 \%$ were assigned to route 2 , and $27 \%$ had a previous history of coronary artery disease. The electrocardiogram was normal or unspecific in $65.5 \%$ of the patients, and $39.4 \%$ of the patients were hypertensive (tab. I). 
The following 383 (36.1\%) patients were excluded from the study: 28 (2.6\%) patients with left bundle-branch block on the electrocardiogram on hospital admission; 81 (7.6\%) patients with type A/B chest pain and depression of the ST segment or inversion of the T wave, or both; 20 (1.8\%) patients with elevation of the ST segment; 164 (15.4\%) patients with acute myocardial infarction; and $90(8.4 \%)$ patients with type D2 chest pain. Six hundred and seventy-seven (63.8\%) patients were eligible for exercise testing according to the protocol, but only 268 (39.5\%) underwent the test, constituting the population studied. The test was not performed in 409 (60.4\%) patients (fig.2), due to some of the following reasons: recurring chest pain, progressive electrocardiographic changes, hemodynamic instability, and instability of the myocardial necrosis markers, not completing the protocol. Some patients did not complete the protocol because of one of the following reasons: learning curve; patients who underwent another method of stratification (myocardial scintigraphy or stress echocardiography); patients unable to walk on the treadmill; and those who did not accept taking part in the study.

The 268 patients undergoing exercise testing were significantly younger $(51.7 \pm 12.1$ years) than those not undergoing the test $(\mathrm{p}=0.0001)$. Most of them were males and they had a higher incidence of dyslipidemia, tobacco use, and familial history of coronary artery disease, and were more frequently assigned to route 3 . Those not undergoing exercise testing were more frequently assigned to route 2 , had a greater incidence of type $\mathrm{A} / \mathrm{B}$ chest pain and a previous history of coronary artery disease (characterizing a group at higher risk). Analysis of the admission electrocardiogram indicated that systemic arterial hypertension and diabetes mellitus were not different between the groups.

\begin{tabular}{|lr|}
\hline \multicolumn{2}{|c|}{$\begin{array}{c}\text { Table I } \text { - Clinical characteristics of the patients initially admitted to the } \\
\text { chest pain } \mathbf{( n = 1 0 6 0 )}\end{array}$} \\
\hline Clinical characteristics & $\mathrm{N}$ \\
\hline Age (mean \pm SD) - years & $59 \pm 15.3$ \\
Male sex & $665(62.7 \%)$ \\
Male sex $\geq 60$ years & $298(28.1 \%)$ \\
\hline Type A chest pain & $224(21.1 \%)$ \\
Type B chest pain & $296(27.9 \%)$ \\
Type C chest pain & $313(29.5 \%)$ \\
Type D chest pain & $227(21.4 \%)$ \\
\hline Normal or unspecific ECG & $734(69 \%)$ \\
ST depression or T-wave inversion ECG & $161(15.1 \%)$ \\
Elevation of the ST segment & $104(9.8 \%)$ \\
LBBB & $28(2.6 \%)$ \\
Pathologic Q wave & $33(3.1 \%)$ \\
\hline Systemic arterial hypertension & $418(39.4 \%)$ \\
Diabetes mellitus & $115(10.8 \%)$ \\
Dyslipidemia & $279(26.3 \%)$ \\
Smoking & $281(26.5 \%)$ \\
Familial history of CAD & $316(29.8 \%)$ \\
Previous AMI & $149(14 \%)$ \\
Use of beta-blockers & $141(13.3 \%)$ \\
& $53(5 \%)$ \\
\hline
\end{tabular}

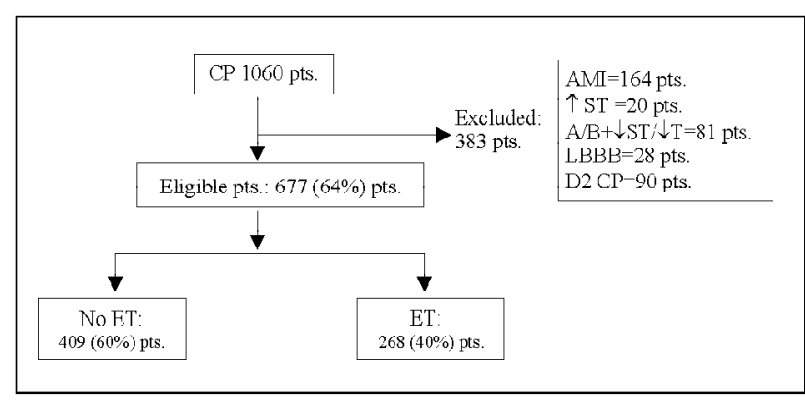

Fig. 2 - Distribution of the patients excluded from the protocol and eligible for ET $\uparrow$ ST- elevation of the ST segment; $\downarrow S T / \downarrow T$-depression of the ST segment or inversion of the $\mathrm{T}$ wave, or both; LBBB- left bundle-branch block; A/B CP - type A or B chest pain.

The characteristics of the patients undergoing exercise testing were as follows: $44(16.4 \%)$ males aged $\geq 60$ years; $186(69.4 \%)$ were assigned to routes $3 / 4 ; 244$ (91\%) patients had a normal or unspecific electrocardiogram on admission; and $154(57.4 \%$ ) patients had type C/D chest pain on admission (tab. II).

The results of the exercise test were as follows: $34(13 \%)$ patients had a positive exercise test, $191(71 \%)$ had a negative exercise test; and $43(16 \%)$ had an inconclusive test.

Correlating the results of the exercise test with the assigned routes, the positive group was observed to have a higher risk on admission; negative results were more frequent in those assigned to routes 3 and 4 (considered having a lower risk on admission) $\mathrm{P}=0.0001$ ) (tab. III).

A significant correlation was observed between the route assigned and the time for performing the test $(\mathrm{p}<0.0001)$, showing that lower-risk patients (routes 3 and 4 ) underwent the test earlier. However, approximately $56 \%$ of the patients following the protocol in the emergency room underwent exercise testing within the first 12 hours after admission, including $27 \%$ of those assigned to route 2 , who were to undergo exercise testing later.

When the results of the exercise test (positive, negative, and inconclusive) were correlated with the clinical and electrocardiographic variables (univariate analysis), we observed that the patients with positive and inconclusive results belonged to a significantly older age group, with a higher incidence of type $\mathrm{A} / \mathrm{B}$ chest pain, of previous coronary artery disease, and were more frequently assigned to route 2 . The patients with positive tests more commonly had diabetes mellitus, and those with negative tests were more frequently assigned to routes 3 and $5(\mathrm{p}<0.0001)$.

The use of beta-blockers was more significant in patients with inconclusive tests $(\mathrm{p}<0.0001)$. However, those with positive tests were receiving more drugs than those with negative tests.

The electrocardiogram on admission showed no statistical significance when correlated with the results of the exercise test; the same occurred with the following variables: systemic arterial hypertension, dyslipidemia, tobacco use, and familial history of coronary artery disease. On the univariate analysis of the results of the test (positive, negative, and inconclusive) with its parameters, we observed a 
Table II - Correlation between the clinical characteristics and the routes assigned to patients eligible for exercise testing, who underwent the test or not (n= 677)

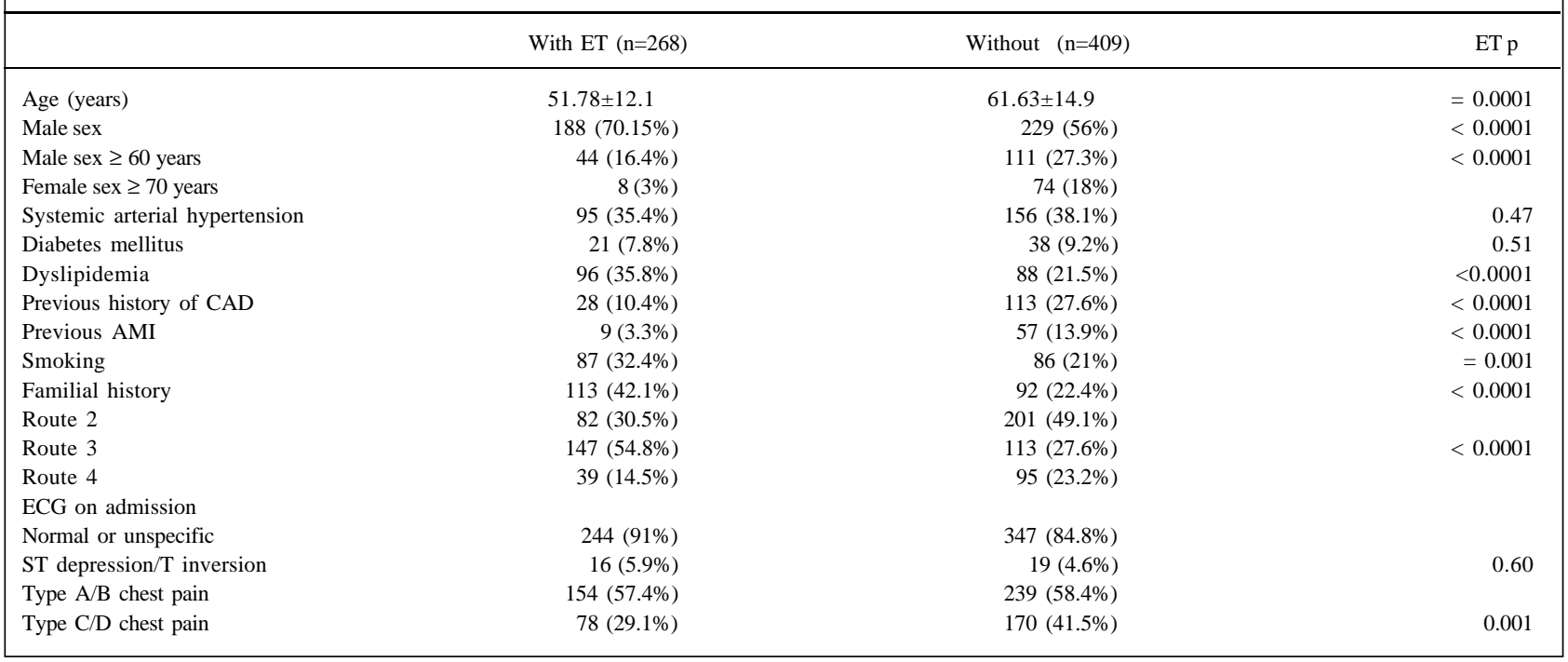

greater proportion of the reduced SBP and hypertensive curves in the group with positive and inconclusive tests, no significant difference being observed between them. The same patients had a mean tolerance to exercise METS (metabolic equivalent), analyzed in the numeric form, significantly lower than that of patients with negative tests. No significant difference was observed between the patients with positive and inconclusive tests. When the double product was analyzed in a numeric form, patients with inconclusive exercise tests were observed to have a mean double product frequently lower than that of patients with positive and negative tests; in the positive test, the double product was lower than that in the negative test (inconclusive<positive $<$ negative $)(\mathrm{p}=0.0001)$.

Of the patients with positive exercise tests, 19 (56\%) had changes in the ST segment during the examination, 12 (35\%) had angina, and $3(9 \%)$ had changes in the ST segment and angina. The time interval between patients' admission to the chest pain unit and performance of the exercise test was significantly greater in patients with positive tests than in those with inconclusive or negative tests $(\mathrm{p}=0.001)$ (tab. IV).

Of the 268 patients undergoing the test, $232(86 \%)$ were discharged right after receiving the test results, and 36 (13\%) were admitted to the hospital due to events or to undergo complementary diagnostic testing.

Of the 34 patients with positive tests, 21 (69\%) were referred for cine coronary angiography, 11 underwent an-

\begin{tabular}{|lccc|}
\hline \multicolumn{4}{|c|}{ Table III - Results of the exercise test and routes assigned } \\
\hline Freq/perc & POS ET (n=34) & NEG ET (n=191) & INC ET (n=43) \\
\hline Route 2 & $18(52.4 \%)$ & $44(23 \%)$ & $20(46.5 \%)$ \\
Route 3 & $13(38.2 \%)$ & $112(58.6 \%)$ & $22(51.1 \%)$ \\
Route 4 & $3(8.8 \%)$ & $35(18.3 \%)$ & $1(2.3 \%)$ \\
$\mathrm{p}=0,0001$ & & & \\
\hline
\end{tabular}

gioplasty, and 2 underwent myocardial revascularization. In that group, 3 patients had normal coronary arteries, and 5 had insignificant coronary artery lesions. Of the 13 patients (38\%) with positive exercise tests who did not undergo cine coronary angiography, 4 underwent myocardial scintigraphy, which was negative for ischemia.

Exercise testing proved to be safe and feasible in patients with chest pain in the emergency room, and no complications were observed during its performance.

\section{Discussion}

Rapid triage and management of patients presenting to the emergency department with chest pain continue to be a great challenge for emergency physicians and cardiologists. The need to rule out acute myocardial infarction in the patient with chest pain has led emergency physicians to admit these patients to the coronary unit, resulting in high costs because of complementary examinations and the hospital stay. However, only 20 to $30 \%$ of these patients are diagnosed with acute coronary artery disease ${ }^{19,20}$. Chest pain units have been developed to assess the diagnosis and to stratify the risk of these patients in the emergency room, using protocols and algorithms that along with noninvasive tests will identify the best management strategy for and profile of these patients, who may be safely discharged with a low rate of cardiac events, therefore reducing the costs of assis tance ${ }^{21}$.

One of the first studies assessing exercise testing in the emergency room in patients with chest pain, showing the feasibility and safety of the method in low-risk individuals, was performed by Tsakonis et $\mathrm{al}^{11}$ in a small group of patients. Those with negative exercise tests were followed up for 6 months, and no events were observed, showing that a negative test in the emergency room may avoid unecessary hospital admissions ${ }^{11}$.

Zalenski et $\mathrm{al}^{22}$, analyzing the sensitivity and specificity 


\begin{tabular}{|c|c|c|c|c|c|c|}
\hline \multicolumn{7}{|c|}{ Table IV - Correlation between the results and the parameters of the exercise test } \\
\hline $\begin{array}{l}\text { Parameters of the ET } \\
\text { Protocol }\end{array}$ & $\begin{array}{l}\text { POS ET } \\
(\mathrm{n}=34)\end{array}$ & $\begin{array}{l}\text { NEG ET } \\
(\mathrm{n}=191)\end{array}$ & $\begin{array}{l}\text { INCET } \\
(\mathrm{n}=43)\end{array}$ & $P$ & Differer & ces \\
\hline Bruce & $16(47 \%)$ & $170(89 \%)$ & $28(65.1 \%)$ & $<0.001$ & $\mathrm{P} \neq \mathrm{N}$ & $\mathrm{P}=\mathrm{I}$ \\
\hline Naughton & $18(52.9 \%)$ & $21(10.9 \%)$ & $15(34.8 \%)$ & & $\mathrm{N} \neq \mathrm{I}$ & \\
\hline SBP Curve Adequate & $19(55.8 \%)$ & $175(91.6 \%)$ & $31(72 \%)$ & $<0.0001$ & $\mathrm{P} \neq \mathrm{N}$ & $\mathrm{P}=\mathrm{I}$ \\
\hline Reduced & $5(14.7 \%)$ & $7(3.6 \%)$ & $8(18.6 \%)$ & & $\mathrm{N} \neq \mathrm{I}$ & \\
\hline Hypertensive & $10(29.4 \%)$ & $9(4.7 \%)$ & $4(9.3 \%)$ & & & \\
\hline Exertion ECG & $19(55.8 \%)$ & $1(0.52 \%)$ & 0 & $<0.0001$ & $\mathrm{P} \neq \mathrm{N}$ & $\mathrm{P} \neq \mathrm{I}$ \\
\hline Angina** & $12(35.2 \%)$ & 0 & 0 & & $\mathrm{~N}=\mathrm{I}$ & \\
\hline Early ET (< 12 h) & $11(32.5 \%)$ & $121(63.3 \%)$ & $18(41.8 \%)$ & $=0.001$ & $\mathrm{P} \neq \mathrm{N} \quad \mathrm{P}=$ & $\mathrm{I} N \neq \mathrm{I}$ \\
\hline Tolerance (METS) * & $7.35 \pm 2.66$ & $11.1 \pm 2.92$ & $8.63 \pm 2.70$ & $=0.0001$ & $\mathrm{P} \neq \mathrm{N} P=$ & $\mathrm{I} N \neq \mathrm{I}$ \\
\hline Double product $*$ & $27217 \pm 7369.69$ & $30612 \pm 6022.69$ & $20870 \pm 5105.80$ & $=0.0001$ & $\mathrm{P} \neq \mathrm{N} \quad \mathrm{P} \neq$ & $\mathrm{I} N \neq \mathrm{I}$ \\
\hline$\Delta \mathrm{T}$ Adm $-\mathrm{ET}^{*}$ & $1445.88 \pm 1103.78$ & $817.95 \pm 611.81$ & $1031.63 \pm 630.60$ & $=0.0001$ & $\mathrm{P} \neq \mathrm{N} \quad \mathrm{P} \neq$ & $\mathrm{I} N=\mathrm{I}$ \\
\hline
\end{tabular}

of each component used in the chest pain unit to assess patients with chest pain, reported that the best values were reached when the CK-MB measurement, the electrocardiogram at rest, and the exercise test were used together, showing the importance of the strategy of approach in this group of patients. Ninety-six patients with chest pain and low risk for acute myocardial infarction were assessed. In the group undergoing exercise testing, $67 \%$ of the patients were negative, $9 \%$ positive, and $24 \%$ inconclusive. The diagnostic protocol including the test showed a $27 \%$ savings compared with the traditional protocol.

Lewis et al ${ }^{13,23}$, using the Bruce treadmill protocol, assessed 93 patients with low-risk chest pain undergoing early exercise testing. Thirteen per cent of the patients had positive tests, and 6 had significant coronary artery disease on coronary angiography. Eighty-seven per cent of the patients had negative or nondiagnostic tests. In the 13-month follow-up, 1 acute myocardial infarction occurred. Later on, that study had its case series extended to include patients with a previous history of coronary artery disease, suggesting that when the maximal heart rate $(>80 \%)$ previewed during the test was reached, it identified the low-risk patients in that group. The same authors, assessing 100 patients with a previous history of coronary artery disease, reported $23 \%$ positive tests, 2 of which had a diagnosis of non- $Q$ infarction. In the 6-month follow-up, no acute myocardial infarction or death of cardiac cause was observed.

Michael et al ${ }^{14}$ assessed 424 patients with intermediaterisk unstable angina randomized for hospitalization and another 212 randomized for the protocol of the observation unit. The protocol group underwent exercise testing, $46 \%$ of the patients had a negative test and were discharged. In the 6month follow-up, in regard to the rate of events, nostatistical significance was observed in any of the groups. The protocol group had a shorter hospital stay at a lower cost.

In our study, of the 1060 patients assisted, 268 (25\%) patients considered at low risk underwent exercise testing. In the study by Polanczyk et al ${ }^{24}$ assessing 276 patients with low-risk chest pain undergoing exercise testing within
48 hours after admission to the emergency room, $27 \%$ of the patients underwent exercise testing. The test was negative in $71 \%$ of the cases, and, in the 6-month follow-up, those patients had a smaller number of aditional visits to the emergency room and a smaller number of readmissions than those with a positive or inconclusive test.

The age bracket of our patients was $51.7 \pm 12.1$ years, constituting an older group than those in other studies of patients with low-risk chest pain; $70 \%$ were males ${ }^{8,13,25}$. Analyzing the group of patients undergoing and not undergoing exercise testing, we observed that the group being tested more frequently had dyslipidemia and a familial history, and smoked, although the previous history of coronary artery disease was significantly greater in the group not tested, $[\mathrm{n}=28(10 \%)]$. The demographic and clinical data in our study showed that the group undergoing exercise testing had a lower risk for events as compared with that of the group not being tested according to NHBL (National Heart, Lung and Blood Institute) criteria ${ }^{26}$. However, when compared with other studies, we observed that that population had a higher risk with a history of previous coronary artery disease of $10 \%{ }^{8,13,25}$.

In regard to the time elapsed between admission and exercise testing, $56 \%$ of the patients underwent the test within less than 12 hours, including 22 (27\%) patients assigned to route 2 (higher risk), who should undergo the test later. This is due to the fact that with the use of systematized programs to rule out acute myocardial infarction and high-risk unstable angina, these patients may have been referred earlier for noninvasive stratification with exercise testing. The study by Lewis et al ${ }^{13}$ used immediate exercise testing (performed within the first hour after admission), but in a population at very low risk. In another study with a low-risk population, the exercise test was performed within 12 hours in $7 \%$ of the patients and between 12 and 24 hours in $45 \%{ }^{24}$.

The time elapsed between admission and exercise testing was greater in the group of patients with positive tests, because most of them were assigned to route 2 , in which the 
test was performed later. In our country, some studies like that of Vivacqua et al presented at the congress of the Sociedade de Cardiologia do Rio de Janeiro in 1998 have shown the use of exercise testing in clinically stable patients with low-risk unstable angina, in whom testing was performed within 24 hours with no complications.

Recently, the ACC/AHA guidelines for unstable angina have recommended that clinically stable patients with low-risk unstable angina undergo stress testing within 12 to 24 hours $^{27}$.

The present study showed that, when the clinical findings were correlated with the results of the positive exercise test, that group was significantly older, had a higher incidence of type A/B chest pain and a history of previous coronary artery disease. Diabetes mellitus has been considered in the literature and in clinical practice as a high-probability predictor of coronary artery disease on admission and has been used as a risk marker in the protocols of chest pain units ${ }^{28}$. In our study, the patients with diabetes mellitus more frequently had a positive exercise test, and this was the only significant clinical marker found.

Of the 268 patients undergoing exercise testing, 34 (13\%) had a positive test, $43(16 \%)$ had inconclusive tests, $191(71 \%)$ had negative tests, and $232(86 \%)$ patients were sent home from the emergency room immediately after receiving the test results. Previous studies have shown a low rate of positive exercise tests, which may range from 0 to $12 \%$ in the low-risk population ${ }^{29}$.

Inconclusive exercise testing continues to be a great challenge in assessing patients with chest pain in the chest pain unit, mainly because it does not safely define the discharge of this group of patients.

In our study, the inconclusive test represented $16 \%$ of the tests performed. Some studies have shown that an inconclusive test relates to a good prognosis in 30 to 180 days ${ }^{30,31}$. The frequency of inconclusive tests has increased in studies. Kirk et $\mathrm{al}^{25}$ reported a frequency of $28 \%$. Dierckes et al. ${ }^{32}$ reported a frequency of $34 \%$, with a rate of events of $3.4 \%$. Many of those patients with a higher probability of coronary artery disease prior to the test and not apt to the test were females, who had a sedentary lifestyle and used medications that changed the heart rate curve.

In our study, most of the patients with inconclusive tests were females on beta-blockers. Inconclusive tests were analyzed along with positive tests, because their prognostic value for cardiac events is still unknown, and patients with high-risk clinical criteria are not identified on admission. In our service, the patients with inconclusive tests are not immediately sent home from the emergency room, because they undergo other complementary examinations, such as imaging tests, before leaving.

Exercise testing is safe, and no complications have occurred during its performance. The safety of the method in the group of patients studied has also been reported in other studies ${ }^{22-24,32}$. Amsterdam et al $^{33}$, studying more than 1000 patients with low-risk chest pain, reported a rate of complication $<1 \%$ during exercise testing.

The results of our study may have implications in clinical practice. The way of approaching patients with chest pain in the emergency room by using a protocol of risk stratification seems to provide more safety to the emergency physician when sending the patient home from the emergency room.

The applicability of this strategy in a higher number of hospitals in Brazil, mainly in public health assistance, is interesting, because the investment needed to start a chest pain unit in an already existing emergency room is not high. The use of a systematic assistance strategy with diagnostic flowchart and trained staff aims at increasing the quality and safety of assistance and reducing hospital costs, as shown in the present study.

\section{References}

1. Hlatky MA. Evaluation of chest pain in the emergency department. N Engl J Med 1997;337:1687-9.

2. Graff LG, Dallara J, Ross MA, et al. Impact on the care of emergency department chest pain patient from the chest pain evaluation registry (CHEPER) study. Am J Cardiol 1997;80:563-8.

3. Laurenti R, Buchalla C, Caratin. Doenças Isquêmicas do Coração. Internações, Tempo de Permanência e Gastos. Brasil, 1993 a 1997. Arq Bras Cardiol, 2000;74: 483-7.

4. Chor D, Fonseca MJ, Andrade CR. Doenças Cardiovasculares: comentários sobre a mortalidade precoce no Brasil. Arq Bras Cardiol 1995;64:15-9.

5. Lee $\mathrm{TH}$, Goldman $\mathrm{L}$. The coronary care unit turns 25 : historical trends and future directions. Ann Intern Med 1998;108:887-94.

6. Pozen MW, D Agostino RB, Mitchel JB, et al. The usefulness of a predictive instrument to reduce inappropriate admissions to the coronary care unit. Ann Intern Med 1980;92:238-42.

7. Ting HH, Lee TH, Soukup JR. Impact of physician experience on triage of emergency room patients with acute chest pain at three teaching hospitals. Ann J Med 1991;91:401-8.

8. Gibler WB, Runyon JP, Levy RC, et al. A rapid diagnostic and treatment center for patients with chest pain in the emergency department. Ann Emerg Med 1995; 25:1-8.
9. Lee TH, Rouan GW, Weisberg MC, et al. Clinical characteristics and natural history of patients with acute myocardial infarction sent home from the emergency room. Ann J Cardiol 1987;60:219-24.

10. Mc Carthy BD, Beshansky JR, Agostino RB, Selker HP. Missed diagnosis of acute myocardial infarction in the emergency department: results from a multicenter study. Ann Emergency Med 1993;22:579-82.

11. Tsakonis JS, Shesser R, Rosenthal R, et al. Safety of immediate treadmill testing in selected emergency department patients with chest pain: a preliminary report. Ann Emerg Med 1991;9:557-9.

12. Kerns JR, Shaub TF, Fontanarosa PB. Emergency cardiac stress testing in the evaluation of emergency department patients with atypical chest pain. Ann Emerg Med 1993;22:794-8.

13. Lewis WR, Amsterdam EA. Utility and safety of immediate exercise testing of low risk patients admitted to the hospital for suspected acute myocardial infarction. Am J Cardiol 1994;74:987-90.

14. Michael E, Farkouh MD, Peter A, Smars MD. The chest pain evaluation in the emergency room (CHEER) investigators. A clinical trial of a chest pain observation unit for patients with unstable angina. N Engl J Med 1998;339: 1882-8.

15. Bassan R, Scofano M, Gamarski R, et al. Dor torácica na sala de emergência: a importância de uma abordagem sistematizada. Arq Bras Cardol 2000;74:13-21. 
16. Bassan R, Pimenta L, Macaciel R, et al. Eficácia de uma estratégia diagnóstica para pacientes com dor torácica e sem supradesnível do segmento ST na sala de emergência. Arq Bras Cardiol 2000;74:405-11.

17. Mason RF, Likar I. Multiple lead exercise electrocardiography. Circulation 1967; 36:517.

18. Bruce RA, et al. Evaluation of functional capacity and exercise tolerance of cardiac patients. Mod Concepts Cardiov Dis 1956;25:321.

19. Goldman L, Cook EF, Brand DA, et al. A computer protocol to predict myocardial infarction in emergency department patients with chest pain. N Engl J Med 1988;318:797-803.

20. Zalenski RJ, Rydman RJ, McCarren M, et al. Feasibility of a rapid diagnostic protocol for an emergency department chest pain unit. Ann Emerg Med 1997;29: 99-108.

21. McNeer JF, Margolis LR, Lee K L, et al. The role of the exercise test in the evaluation of patients for ischemic heart disease. Circulation 1978;57:64-70

22. Zalenski RJ, McCarren M, Roberts R, et al. Evaluation of a chest pain diagnostic protocol to exclude acute cardiac ischemia in the emergency department. Arch Intern Med 1997; 157:1085-91

23. Lewis WR, Amsterdam EA, Turnipseed S, et al. Immediate exercise testing of low risk patients with known coronary artery disease presenting to the emergency department with chest pain. J Am Coll Cardiol 1999;33:1843-7.

24. Polanczyk CA, Johnson PA, Lee TH, et al. Clinical correlates and prognostic significance of early negative exercise tolerance test in patients with acute chest pain seen in the hospital emergency department. J Am Coll Cardiol 1998;81:287-92.
25. Kirk JD, Turnipseed S, Lewis WR, Amsterdam EA. Evaluation of chest pain in low-risk patients presenting to the emergency department: the role of immediate exercise testing. Ann Emerg Med 1998;32:1-7.

26. Brauwald E, Mark DB, Jones RH, et al. Unstable angina - Diagnoses and management clinical practice guideline. Agency for Healthcare Policy and Research and National Heart Lung and Blood Institute, Public Health Service vs Department of Health and Human Services AH CPR; 154;1994:28-92

27. Brauwald E, MD, FACC, chair. ACC/AHA Guidelines for the management of patients with unstable angina and non-ST segment elevation myocardial infarction. J Am Coll Cardiol 2000;36:970-1062.

28. Lee TH, Cook F, Goldman L, et al. Acute chest pain in the emergency room. Identification and examination of low-risk patients. Arch Intern Med 1985;145.

29. Christopher R, Defilippi MD, Marshall S Runge. Avaliação do paciente com dor torácica. Clínicas Cardiológicas da América do Norte 1999;17.

30. Abreo G, Rohit P, Dong Z, et al. Stress testing markedly underestimates the prevalence of coronary artery disease in a "low risk" chest pain unit: results of a prospective randomized trial. Circulation 1997;96:1-272.

31. Gomez MA, Anderson JL, Karagounis LA, Muhlestein JB. An emergency department based protocol for rapidly ruling out ischemia reduces hospital time and expense: results of a randomized study (Romio). J Am Coll Cardiol 1996; 28: 25-31.

32. Dierckes DB, Gibler WB, Tiepu L, Sayre MR, Storrow AB. Identification of patients at risk by emergency department chest pain center. Am J Cardiol 2000; 86: 289-92.

33. Amsterdan E, Kirk JD, Lewis WR. Immediate exercise stress testing for assessment of clinical risk in patients presenting to the emergency department with chest pain: results in over 1000 patients (abst). Circulation 1998; 98(Suppl I): 1-774 